\title{
Anti-Mullerian hormone (AMH) as predictor of ovarian reserve
}

\author{
Chaitanya A. Shembekar ${ }^{1}$, Jayshree J. Upadhye ${ }^{1 *}$, \\ Manisha C. Shembekar ${ }^{2}$, Shravani H. Welekar ${ }^{3}$
}

${ }^{1}$ Department of Obstetrics and Gynecology, ${ }^{2}$ Department of Anaesthesia, ${ }^{3}$ Department of Embryology, Omega Hospital, Nagpur, Maharashtra, India

Received: 01 July 2017

Accepted: 29 July 2017

\section{*Correspondence:}

Dr. Jayshree J. Upadhye,

E-mail: jayshreeupadhye@gmail.com

Copyright: (c) the author(s), publisher and licensee Medip Academy. This is an open-access article distributed under the terms of the Creative Commons Attribution Non-Commercial License, which permits unrestricted non-commercial use, distribution, and reproduction in any medium, provided the original work is properly cited.

\section{ABSTRACT}

Background: Anti-Müllerian hormone $(\mathrm{AMH})$ is produced by the granulosa cells of preantral and small antral follicles and its levels can be assessed in serum. Since the number of ovarian follicles declines with increasing age, AMH levels might be used as a marker for ovarian ageing. Therefore, we studied the relationship between AMH levels and ovarian response during ovarian stimulation for In vitro fertilization.

Methods: A total of 100 patients who have undergone their ICSI treatment cycle using a GnRH antagonist protocol were retrospectively included. Co-relation between AMH and antral follicular count (AFC) was assessed.

Results: In present study, 36\% patients had normal $\mathrm{AMH}, 18 \%$ patients were in low normal range, $5 \%$ patients had low values and $2 \%$ patients had very low values. $41 \%$ of patients had values in high range suggestive of PCOS. Amongst this, $21 \%$ had values between 4 to $8 \mathrm{ng} / \mathrm{ml}$ where we got good AFC count and good result in terms of pregnancy. $80 \%$ were good responders while $20 \%$ were poor responders. When we evaluated the relationship of retrieved oocyte counts with the parameters included, we found that only basal AMH levels and the number of antral follicles were statistically correlated.

Conclusions: High AMH levels correlated with low cancellation rates, retrieval of more eggs, higher live birth rates and a high chance for freezing of embryos. Low AMH levels (alone) do not predict low success rates in women under 35 years of age.

Keywords: Anti-mullerian hormone, Antral follicular count, Oocytes

\section{INTRODUCTION}

Ovarian reserve is defined as the functional potential of the ovary, and reflects the number and quality of the oocytes in the ovary at any given time. ${ }^{1}$

With the understanding that chronological age alone is an inadequate predictor of the ovarian reserve, multiple tests have been developed to assess ovarian function (i.e., "ovarian reserve" tests). Some of these tests include basal FSH, basal inhibin B, the clomiphene citrate (CC) challenge test, basal $\mathrm{E}_{2}$, the $\mathrm{GnRH}$ challenge test, the ovarian antral follicle count (AFC) as assessed by transvaginal ultrasound examination, and serum levels of anti-Müllerian hormone (AMH). Although such tests are frequently labeled ovarian reserve tests, they are more accurately ovarian response tests. ${ }^{2}$

Anti-Müllerian hormone (AMH) is produced by the granulosa cells of preantral and small antral follicles and its levels can be assessed in serum. Since the number of ovarian follicles declines with increasing age, AMH levels might be used as a marker for ovarian ageing. ${ }^{3}$

Human female serum contains measurable amounts of AMH during the reproductive life span. ${ }^{3}$ Since AMH is 
solely produced in the growing ovarian follicles, serum levels may be used as a marker for ovarian reserve, representing the quantity and quality of the ovarian follicle pool. ${ }^{3}$ The ovarian reserve, constituted by the size of the ovarian follicle pool and the quality of the oocytes therein, declines with increasing age, resulting in the decrease of a woman's reproductive function. ${ }^{3}$

Anti-Müllerian hormone seems to be the best endocrine marker for assessing the age-related decline of the ovarian pool in healthy women; thus, it has a potential ability to predict future reproductive lifespan. The most established role for AMH measurements is before in vitro fertilization is initiated, because AMH can be predictive of the ovarian response, namely poor and hyperresponses. $^{4}$

Plasma AMH assessments are superior to FSH in identifying women with reduced ovarian reserve. ${ }^{4}$ Antimüllerian hormone assessment should be considered as a useful adjunct to FSH/oestradiol levels and antral follicle count when estimating ovarian reserve. ${ }^{5}$

It is widely accepted that the reduction of AMH levels in serum is the first indication of a decline in the follicular reserve of the ovaries. ${ }^{6}$ AMH concentration remains stable throughout the menstrual cycle. ${ }^{6}$ In conditions with high LH and normal or low FSH levels, as in PCOS, AMH concentrations are positively correlated with $\mathrm{LH}$ concentrations, while they are not negatively correlated with FSH. ${ }^{6}$

In a normal ovary, AMH works to slow and prevent the premature development of the follicles before they are mature - keeping the ovary from developing eggs prematurely. $^{7}$

Present study was undertaken to determine the predictive value of antimüllerian hormone $(\mathrm{AMH})$ as a marker for ovarian reserve.

\section{METHODS}

It was a retrospective analysis carried out at in vitro fertilization (IVF) clinic. A total of 100 patients who have undergone their ICSI treatment cycle using a GnRH antagonist protocol were retrospectively included.

All patients recruited for ICSI, irrespective of indications, were included in the study. On day 2 of a spontaneous cycle, patients underwent a transvaginal ultrasound examination to assess the number of antral follicles, measuring 2-5 $\mathrm{mm}$ and endometrial thickness. Patients with thin endometrium were recruited. On the same day a venous blood sample was obtained for the measurement of AMH, FSH, estradiol (E2) and inhibin B

Ovulation induction was initiated with recombinant FSH, 150-300 IU/day, depending on AMH, age and AFC. After more than three follicles larger than $18 \mathrm{~mm}$ were observed, 250 microgram of recombinant hCG was administered subcutaneously and 35 hours later oocyte retrieval was done under general anesthesia.

The study group was divided into two subgroups according to the number of oocytes retrieved. Patients with an oocyte count of five or more were considered good responders, and patients with less than five oocytes as poor responders. ICSI (intracytoplasmic sperm injection) was done in all patients.

The transfer of the embryos was performed 48 hours after the procedure. Four cell grade 1 embryos were transferred. A maximum of three embryos were transferred. On the 12th to 14th days of the transfer, a serum $\beta$-hCG test was performed to confirm pregnancy. To support the luteal phase, micronized progesterone was given.

The main outcome measures of the study were the number of oocytes retrieved i.e. ovarian response to stimulation. As described previously, poor response was defined as fewer than 4 oocytes obtained.

\section{RESULTS}

Maximum patients i.e. $42 \%$ patients were between 31-35 years. $29 \%$ patients were between $26-30$ years, $16 \%$ patients were between $36-40$ years, $12 \%$ patients were between $21-25$ years while only $1 \%$ of patients were above 40 years of age.

Table 1: Age group.

\begin{tabular}{|lll|}
\hline Age group & No. of patients & Percent \\
\hline 21-25 years & 12 & 12 \\
\hline 26-30 years & 29 & 29 \\
\hline $31-35$ years & 42 & 42 \\
\hline $36-40$ years & 16 & 16 \\
\hline$>40$ years & 1 & 1 \\
\hline
\end{tabular}

Table 2: Type of infertility.

\begin{tabular}{|lll|}
\hline Type of infertility & No. of patients & Percent \\
\hline Primary & 74 & 74 \\
\hline Secondary & 26 & 26 \\
\hline
\end{tabular}

In present study, $74 \%$ of patients had primary infertility while $26 \%$ of patients had secondary infertility.

Table 3: Indication for ICSI.

\begin{tabular}{|lll|}
\hline Indication for ICSI & No. of patients & $\%$ \\
\hline Tubal factor & 22 & 22 \\
\hline Male factor & 20 & 20 \\
\hline PCOS & 17 & 17 \\
\hline Endometriosis & 5 & 5 \\
\hline Unexplained infertility & 18 & 18 \\
\hline More than 1 factor & 18 & 18 \\
\hline
\end{tabular}


In present study, $22 \%$ of patients had tubal factor, $20 \%$ of patients had male factor, $17 \%$ of patients had polycystic ovarian syndrome (PCOS) while $5 \%$ patients had endometriosis. $18 \%$ of patients had unexplained infertility.

Table 4: Number of ICSI cycle.

\begin{tabular}{|lll|}
\hline No. of ICSI cycle & No. of patients & $\%$ \\
\hline First & 93 & 93 \\
\hline Second & 3 & 3 \\
\hline Third & 2 & 2 \\
\hline Fourth & 2 & 2 \\
\hline
\end{tabular}

In present study, $93 \%$ of patients had their first cycle of ICSI, 3\% of patients had their second cycle of ICSI, $2 \%$ of patients had third cycle of ICSI and $2 \%$ of patients had their fourth cycle of ICSI.

Table 5: AMH levels.

\begin{tabular}{|llll|} 
& AMH levels & $\begin{array}{l}\text { No. of } \\
\text { patients }\end{array}$ & $\%$ \\
\hline High & Over $4.0 \mathrm{ng} / \mathrm{ml}$ & 41 & 41 \\
\hline Normal & $1.5-4.0 \mathrm{ng} / \mathrm{ml}$ & 36 & 36 \\
\hline $\begin{array}{l}\text { Low normal } \\
\text { range }\end{array}$ & $1.0-1.5 \mathrm{ng} / \mathrm{ml}$ & 18 & 18 \\
\hline Low & $0.5-1.0 \mathrm{ng} / \mathrm{ml}$ & 5 & 5 \\
\hline Very low & Less than $0.5 \mathrm{ng} / \mathrm{ml}$ & 2 & 2 \\
\hline
\end{tabular}

In present study, 36\% patients had normal AMH, $18 \%$ patients were in low normal range, $5 \%$ patients had low values and $2 \%$ patients had very low values. $41 \%$ of patients had values in high range suggestive of PCOS. Amongst this, $21 \%$ had values between 4 to $8 \mathrm{ng} / \mathrm{ml}$ where we got good AFC count and good result in terms of pregnancy.

Table 6: Ovarian response.

\begin{tabular}{|lll|}
\hline Ovarian response & No. of patients & $\%$ \\
\hline Good responders & 80 & 80 \\
\hline Poor responders & 20 & 20 \\
\hline
\end{tabular}

The study group was divided into two subgroups according to the number of oocytes retrieved. Patients with an oocyte count of five or more were considered good responders, and patients with less than five as poor responders. In present study, $80 \%$ were good responders while $20 \%$ were poor responders.

Table 7: Pregnancy outcome.

\begin{tabular}{|lll|}
\hline Pregnancy outcome & No. of patients & $\%$ \\
\hline Total no. of pregnancies & 54 & 54 \\
\hline Triplets & 3 & 3 \\
\hline Twins & 7 & 7 \\
\hline Abortion & 10 & 10 \\
\hline Biochemical pregnancies & 8 & 8 \\
\hline
\end{tabular}

In present study, fifty four patients conceived. Three patients had triplets, seven of them continued as twin pregnancies, and forty four patients as singleton pregnancy. Ten pregnancies ended up in abortion and eight were biochemical pregnancies.

When we evaluated the relationship of retrieved oocyte counts with the parameters included, we found that only basal AMH levels and the number of antral follicles correlated well.

\section{DISCUSSION}

Maximum patients i.e. $42 \%$ patients were between 31-35 years. $29 \%$ patients were between $26-30$ years, $16 \%$ patients were between $36-40$ years, $12 \%$ patients were between $21-25$ years while only $1 \%$ of patients were above 40 years of age. Patients with low and very low values of AMH were above 35 years of age.

$\mathrm{K}$. Hansen et al found that even after correcting for chronological age, two of the tests, the ovarian AFC and serum levels of $\mathrm{AMH}$, were still significantly correlated with the ovarian primordial follicle number. In addition, approximately $74 \%$ of the variation in ovarian primordial follicle count could be explained with only two of the parameters, chronological age and the ovarian AFC. 2 Kelton $\mathrm{P}$ et al found that plasma $\mathrm{AMH}$ levels remained relatively static (20-25 pmol/L) from 18 to 29 years of age. By 30 years of age, plasma AMH levels start to drop rapidly, reaching only $10 \mathrm{pmol} / \mathrm{L}$ by 37 years..$^{5}$

In present study, $74 \%$ of patients had primary infertility while $26 \%$ of patients had secondary infertility. $93 \%$ of patients had their first cycle of ICSI, 3\% of patients had their second cycle of ICSI, $2 \%$ of patients had third cycle of ICSI and $2 \%$ of patients had their fourth cycle of ICSI.

In present study, $36 \%$ patients had normal AMH, $18 \%$ patients were in low normal range, $5 \%$ patients had low values and $2 \%$ patients had very low values. $41 \%$ of patients had values in high range suggestive of PCOS. Amongst this, $21 \%$ had values between 4 to $8 \mathrm{ng} / \mathrm{ml}$ where we got good AFC count and good result in terms of pregnancy.

The study group was divided into two subgroups according to the number of oocytes retrieved. Patients with an oocyte count of five or more were considered good responders, and patients with less than five as poor responders. In present study, $80 \%$ were good responders while $20 \%$ were poor responders. When we evaluated the relationship of retrieved oocyte counts with the parameters included, we found that only basal AMH levels and the number of antral follicles were statistically correlated.

Kelton $\mathrm{P}$ et al found that using a cut off value of 8.1 $\mathrm{pmol} / \mathrm{L}$, plasma AMH assessment could predict poor ovarian reserve on a subsequent IVF cycle with a 
sensitivity of $80 \%$ and a specificity of $85 \% .^{5}$ Kelton $\mathrm{P}$ et al found that plasma $\mathrm{AMH}$ assessments are superior to FSH in identifying women with reduced ovarian reserve. Anti-müllerian hormone assessment should be considered as a useful adjunct to FSH/oestradiol levels and antral follicle count when estimating ovarian reserve. ${ }^{5}$

Fiçicioğlu $\mathrm{C}$ et al found that patients with fewer than five retrieved oocytes had lower day $3 \mathrm{AMH}$ levels, fewer antral follicles, and lower hCG day E2 levels. Thus, basal antral follicle count and basal AMH levels are good tools for use in counseling patients. ${ }^{8} \mathrm{C}$ Fiçicioğlu et al found that levels of $\mathrm{AMH}$ would predict the number of oocytes with a positive predictive rate of $96 \%$, although it had little value for predicting pregnancy. $8 \mathrm{C}$ Fiçicioğlu et al revealed that the most sensitive and specific indicator of ovarian reserve is the level of $\mathrm{AMH}$, it does not indicate pregnancy success as well when $0.25 \mathrm{pg} / \mathrm{mL}$ is taken as a cut-off value. ${ }^{8}$ Fiçicioğlu $\mathrm{C}$ et al demonstrated an association between early follicular phase serum AMH and number of retrieved oocytes despite clinically similar day. ${ }^{8}$ Fiçicioğlu $\mathrm{C}$ et al measured serum FSH and E2 levels in patients of all age groups. Baseline FSH, LH, and E2 levels are good predictors of ovarian reserve. ${ }^{8} \mathrm{C}$ Fiçicioğlu et al found that basal antral follicle count is correlated but weakly with the number of retrieved oocytes during assisted reproduction cycles. ${ }^{8}$ Fiçicioğlu $\mathrm{C}$ et al found that AMH was the best indicator of ovarian reserve with a high sensitivity and specificity. ${ }^{8}$

Better AMH than FSH specificity has been previously demonstrated and is also supported by Fiçicioğlu $\mathrm{C}$ et al. AMH $<1.05 \mathrm{ng} / \mathrm{mL}$, however, does not define DOR. It only defines DOR with significantly decreased live-birth chances. It also does not warrant withholding of treatment because even DOR patients with very low to undetectable AMH still achieve rather surprising livebirth rates. ${ }^{8}$

Van Rooij et al found that serum AMH levels were highly correlated with the number of antral follicles $(\mathrm{r}=$ $0.77 ; \mathrm{P}<0.01)$ and the number of oocytes retrieved $(\mathrm{r}=$ $0.57, \mathrm{P}<0.01){ }^{9}$ Van Rooij et al combined antral follicle count with AMH and inhibin B to provide for better prediction. ${ }^{9,12}$ Dillon $\mathrm{K}$ et al found that participants with a pre-treatment $\mathrm{AMH}$ level $>2 \mathrm{ng} / \mathrm{mL}$ recovered at a rate of $11.9 \%$ per month after chemotherapy, whereas participants with pre-treatment AMH levels $\leq 2 \mathrm{ng} / \mathrm{mL}$ recovered at a rate of $2.6 \%$ per month after therapy. ${ }^{10}$

Akira Ivase et al found that the median AMH level was $2.98 \mathrm{ng} / \mathrm{mL}$ and $3.92 \mathrm{ng} / \mathrm{mL}$ before operation and was significantly reduced to a median level of $2.24 \mathrm{ng} / \mathrm{mL}$ and $3.29 \mathrm{ng} / \mathrm{mL}$ at 1 month after operation in the endometrioma group $(n=29)$ and the non-endometrioma group $(n=21)$, respectively. ${ }^{11}$ Kelton $\mathrm{T}$ found that Serum $\mathrm{AMH}$ is a sensitive marker of age-related decline in ovarian reserve status. A serum AMH result $>36$ pmol $\mathrm{L}-1$, or above the 75th percentile for age, is highly suggestive of a diagnosis of PCOS. A serum AMH result below the $10^{\text {th }}$ percentile for age suggests accelerated loss of ovarian reserve, while an AMH result exceeding 20 pmol L-1 suggests an increased risk of OHSS during IVF treatment. ${ }^{12}$ Brodin $\mathrm{T}$ et al found that all ORTs correlated significantly with each other, with the strongest correlation between AFC and AMH $(r=0.71, p$ $<0.0001)$. Univariately, $\mathrm{AMH}$ and age equivalently predicted live birth (c-statistic 0.61), and together they provided a significantly better model (c-statistic 0.64). For prediction of poor and excessive response the best model included AMH, AFC and age (c-statistic 0.89). ${ }^{13}$

Lauren $\mathrm{Z}$ et al found that among 97 women who underwent AMH testing, 32 (33.0\%) had elevated AMH levels. Hyperandrogenism was reported by $8(25.0 \%)$ women with elevated $\mathrm{AMH}$ and none with $\mathrm{AMH}$ concentrations lower than $4.7 \mathrm{ng} / \mathrm{mL} \quad(\mathrm{P}<0.001)$. Irregular menstrual cycles before hormonal contraceptive use were reported by $16(24.6 \%)$ of 65 women with AMH concentrations lower than $4.7 \mathrm{ng} / \mathrm{mL}$ and 11 $(34.4 \%)$ with elevated AMH $(\mathrm{P}=0.34)$. Of the 20 women with elevated $\mathrm{AMH}$ who returned for further evaluation, $16(80.0 \%)$ had polycystic ovaries and $13(65.0 \%)$ were diagnosed with PCOS (Rotterdam criteria). ${ }^{14}$

\section{CONCLUSION}

When basal AMH levels and the number of antral follicles were correlated, high AMH levels correlate with low cancellation rates, retrieval of more eggs, higher live birth rates and a high chance for freezing of embryos. Low AMH levels (alone) do not predict low success rates in women less than 35 years of age. Couples should not be excluded from attempting assisted reproductive cycles due to low AMH values alone because live birth success rates were reasonable in these cases.

Funding: No funding sources Conflict of interest: None declared

Ethical approval: The study was approved by the Institutional Ethics Committee

\section{REFERENCES}

1. Chang HJ, Han SH, Lee JR, Jee BC, Lee BI, Suh CS, Kim SH. Impact of laparoscopic cystectomy on ovarian reserve: serial changes of serum antiMüllerian hormone levels. Fertil Steril. 2010;94(1):343-9.

2. Hansen KR, Hodnett GM, Knowlton N, Craig LB. Correlation of ovarian reserve tests with histologically determined primordial follicle number. Fertility and sterility. 2011 Jan 31;95(1):170-5.

3. Visser JA, de Jong FH, Laven JS, Themmen AP. Anti-Müllerian hormone: a new marker for ovarian function. Reproduction. 2006 Jan 1;131(1):1-9.

4. Grynnerup AG, Lindhard A, Sørensen S. The role of anti-Müllerian hormone in female fertility and infertility - an overview. Acta Obstet Gynecol Scand. 2012;91:1252-60. 
5. Tremellen KP, Kolo M, Gilmore A, Lekamge DN. Anti- müllerian hormone as a marker of ovarian reserve. Aust N Z J Obstet Gynecol. 2005;45(1):204.

6. Karkanaki A, Vosnakis C, Panidis D. The clinical significance of anti-Müllerian hormone evaluation in gynecological endocrinology. Hormones. 2011;10(2):95-103.

7. Fiona McCulloch AMH. An Important Hormone Test for Women with PCOS, White lotus Integrative Medicine. 2015

8. Fiçicioğlu C, Kutlu T, Baglam E, Bakacak Z. Early follicular antimüllerian hormone as an indicator of ovarian reserve. Fertil Sterility. 2006;85(3):592-6.

9. Rooij V, Broekmans F. Serum anti-Müllerian hormone levels: a novel measure of ovarian reserve. Hum Reprod. 2002;17(12):3065-71.

10. Dillon KE, Sammel MD, Prewitt M, Ginsberg JP, Walker D, Mersereau JE, Gosiengfiao Y, Gracia CR. Pretreatment antimüllerian hormone levels determine rate of posttherapy ovarian reserve recovery: acute changes in ovarian reserve during and after chemotherapy. Fertil Steril. 2013;99(2):477-83.

11. Iwase A, Hirokawa W, Goto M, Takikawa S, Nagatomo Y, Nakahara T et al. Serum anti-Müllerian hormone level is a useful marker for evaluating the impact of laparoscopic cystectomy on ovarian reserve. Fertil Steril. 2010;94(7):2846-9.

12. Tremellen K, Zander- Fox D. Serum anti- Mullerian hormone assessment of ovarian reserve and polycystic ovary syndrome status over the reproductive lifespan. Aust N Z J Obstet Gynecol. 2015;55(4):384-9.

13. Brodin T, Hadziosmanovic N, Berglund L, Olovsson M, Holte J. Comparing four ovarian reserve markers-associations with ovarian response and live births after assisted reproduction. Acta Obstetricia et Gynecologica Scandinavica. 2015;94(10):1056-63.

14. Safier LZ, Grossman LC, Chan CW, Sauer MV, Lobo RA, Douglas NC. Use of anti- Müllerian hormone testing during ovarian reserve screening to identify women at risk of polycystic ovary syndrome. Int J Gynecol Obstet. 2016;135(1):73-6.

Cite this article as: Shembekar CA, Upadhye JJ, Shembekar MC, Welekar SH. Anti-Mullerian hormone $(\mathrm{AMH})$ as predictor of ovarian reserve. Int $\mathbf{J}$ Reprod Contracept Obstet Gynecol 2017;6:4006-10. 